\title{
ON SOME FACTORIAL PROPERTIES OF SUBRINGS
}

\author{
By Piotr Jędrzejewicz, Łukasz Matysiak and Janusz Zieliński
}

\begin{abstract}
We discuss various factorial properties of subrings as well as properties involving irreducible elements and square-free elements, in particular ones connected with Jacobian conditions.
\end{abstract}

Introduction. Throughout the paper, by a ring we mean a commutative ring with unity. By a domain we mean a (commutative) ring without zero divisors. By $R^{*}$ we denote the set of all invertible elements of a ring $R$. If $R$ is a domain, then by $R_{0}$ we denote its field of fractions. If elements $a, b \in R$ are associated in a ring $R$, we write $a \sim_{R} b$. We write $\left.a\right|_{R} b$ if $b$ is divisible by $a$ in $R$. Furthermore, we write $a \operatorname{rpr}_{R} b$ if $a$ and $b$ are relatively prime in $R$, that is, have no common non-invertible divisors. We use a subscript indicating the ring when we compare properties in a ring $A$ and in its subring $R$. If $R$ is a ring, then by $\operatorname{Irr} R$ we denote the set of all irreducible elements of $R$, and by $\operatorname{Sqf} R$ we denote the set of all square-free elements of $R$, where an element $a \in R$ is called square-free if it cannot be presented in the form $a=b^{2} c$ with $b \in R \backslash R^{*}, c \in R$.

Now, let $A=k\left[x_{1}, \ldots, x_{n}\right]$ be the algebra of polynomials over a field $k$ of characteristic zero. Let $f_{1}, \ldots, f_{r} \in A$ be algebraically independent over $k$, where $r \in\{1, \ldots, n\}$. Let $R=k\left[f_{1}, \ldots, f_{r}\right]$. By $\operatorname{jac}_{x_{i_{1}}, \ldots, x_{i_{r}}}^{f_{1}, \ldots, f_{r}}$ we denote the Jacobian determinant of $f_{1}, \ldots, f_{r}$ with respect to $x_{i_{1}}, \ldots, x_{i_{r}}$. We recall from $\mathbf{1 7}$ the following generalization of the Jacobian Conjecture.

ConjeCture. If $\operatorname{gcd}\left(\operatorname{jac}_{x_{i_{1}}, \ldots, x_{i_{r}}}^{f_{1}, \ldots, f_{r}}, 1 \leqslant i_{1}<\ldots<i_{r} \leqslant n\right) \in k \backslash\{0\}$, then $R$ is algebraically closed in $A$.

Note that by Nowicki's characterization the above assertion means that $R$ is a ring of constants of some $k$-derivation of $A$ ([19], Theorem 5.4, [18],

2010 Mathematics Subject Classification. Primary 13F20, Secondary 14R15.

Key words and phrases. Irreducible element, square-free element, factorization, Jacobian Conjecture. 
Theorem 4.1.4, $[\mathbf{6}], 1.4)$. Note also that case $r=1$ is true $([\mathbf{2}]$, Proposition 14, see also 14, Proposition 4.2). Moreover, we refer the reader to van den Essen's book $[\mathbf{7}]$ for information on the Jacobian Conjecture.

Generalizing the results of $[\mathbf{1 6}]$ and $[\mathbf{4}]$, the above gcd condition was expressed in terms of irreducible and square-free elements.

Theorem $([\mathbf{1 7}], 2.4)$ The following conditions are equivalent:

(i) $\operatorname{gcd}\left(\operatorname{jac}_{x_{i_{1}}, \ldots, x_{i_{r}}}^{f_{1}, \ldots, f_{r}}, 1 \leqslant i_{1}<\ldots<i_{r} \leqslant n\right) \in k \backslash\{0\}$,

(ii) $\operatorname{Irr} R \subset \operatorname{Sqf} A$,

(iii) $\operatorname{Sqf} R \subset \operatorname{Sqf} A$.

Finally recall that condition (iii) is in a much more general case equivalent to some factoriality property.

Theorem $([\mathbf{1 7} \mid, 3.4)$ Let $A$ be a unique factorization domain. Let $R$ be a subring of $A$ such that $R^{*}=A^{*}$ and $R_{0} \cap A=R$. The following conditions are equivalent:

(i) $\operatorname{Sqf} R \subset \operatorname{Sqf} A$,

(ii) for every $x \in A$, $y \in \operatorname{Sqf} A$, if $x^{2} y \in R \backslash\{0\}$, then $x, y \in R$.

A subring $R$ satisfying the above condition (ii) is called square-factorially closed in $A$ ([17], Definition 3.5). Under the assumptions of Theorem 3.4, square-factorially closed subrings are root closed $([\mathbf{1 7}]$, Theorem 3.6), see [1] and [5] for information on root closed subrings.

The above theorem corresponds with the known fact that a subring $R$ of a UFD $A$ such that $R^{*}=A^{*}$ is factorially closed in $A$ if and only if $\operatorname{Irr} R \subset$ $\operatorname{Irr} A$. Recall that a subring $R$ of $A$ is called factorially closed iff for every $x, y \in A, x y \in R \backslash\{0\}$ implies $x, y \in R$. Rings of constants of locally nilpotent derivations in domains of characteristic zero are factorially closed (see 8 and 6 for details). In a more general setting, with respect to the multiplicative structure only, a submonoid satisfying such a condition is called divisor-closed (see 9 for details).

A general question stated in [17], when conditions (ii) or (iii) of Theorem 2.4 imply algebraic closedness of $R$ in $A$, inspired us to study their relations with other conditions of this type (see Proposition 3.3 below). Theorem 3.4 above motivates us to investigate in Section 4 various properties having a form of factoriality, in particular those similar to (ii).

The aim of this paper is to collect or develop various conditions related to the ones above. Of course, the general question stated in the mentioned conjecture is very difficult. This is why it may be helpful to study similar properties first. 
1. Divisibility, relative primeness, etc. in a subring. In this section we describe relationships between various conditions on a subring of a domain of arbitrary characteristic.

If $R$ is a subring of a domain $A$, the condition

$$
R_{0} \cap A=R
$$

appears in many situations, the most important example being Hilbert's 14th Problem. It appears also in characterizations of rings of constants of derivations ([19], Theorem 5.4, [18], Theorem 4.1.4, [10], Theorem 1.1, [11], Theorem 2.5), as well as in assumptions of theorems concerning necessary or sufficient conditions for $p$-bases of such rings ([12], Theorems 1.4, 1.5, 15], Propositions 2.1, 2.2). According to the multiplicative structure, a submonoid of a (commutative cancellative) monoid satisfying the above condition is called saturated ([9], Definition 2.4.1, p. 68, Corollary 2.4.3, p. 70).

Proposition 1.1. Let $A$ be a domain, and $R$ be a subring of $A$. Then the following conditions are equivalent:

(i) $R_{0} \cap A=R$,

(ii) $R=L \cap A$ for some subfield $L \subset A_{0}$,

(iii) for every $a \in R, b \in A$, if $a b \in R \backslash\{0\}$, then $b \in R$,

(iv) for every $a, b \in R$, if $\left.a\right|_{A} b$, then $\left.a\right|_{R} b$.

Proof. (i) $\Leftrightarrow$ (iv) Obviously, $R \subset R_{0} \cap A$. Thus the equality $R_{0} \cap A=R$ is equivalent to the inclusion $R_{0} \cap A \subset R$, the latter meaning that for arbitrary $a, b \in R, a \neq 0$, if $\frac{b}{a} \in A$, then $\frac{b}{a} \in R$, which is statement (iv).

(i) $\Rightarrow$ (ii) Put $L=R_{0}$.

(ii) $\Rightarrow$ (i) If $R=L \cap A$, then $R \subset L$, and since $L$ is a field, we have $R_{0} \subset L$. Hence, $R_{0} \cap A \subset L \cap A=R$. The opposite inclusion is evident.

(iii) $\Rightarrow$ (iv) Let $a, b \in R$. If $b=0$, then obviously $\left.a\right|_{R} b$. Let $b \neq 0$. If $\left.a\right|_{A} b$, then $b=a c$ for some $c \in A$. By (iii) we have $c \in R$, and consequently $\left.a\right|_{R} b$.

(iv) $\Rightarrow$ (iii) If $a b \in R \backslash\{0\}$ for $a \in R, b \in A$, then $\left.a\right|_{A} a b$. Therefore, $\left.a\right|_{R} a b$, by (iv). Hence $a b=a r$ for some $r \in R$. If $a \neq 0$, then $b=r \in R$, since $A$ is a domain. If $a=0$, then $a b=0$, contrary to $a b \in R \backslash\{0\}$.

Proposition 1.2. Let $A$ be a domain, and $R$ be a subring of $A$. Then the following conditions are equivalent:

(i) $R^{*}=A^{*} \cap R$,

(ii) for every $a \in R$, if $a \in A^{*}$, then $a \in R^{*}$,

(iii) for every $a, b \in R$, if $a \operatorname{rpr}_{A} b$, then $a \operatorname{rpr}_{R} b$. 
Proof. (i) $\Leftrightarrow$ (ii) Clearly $R^{*} \subset A^{*} \cap R$. Therefore, the equality $R^{*}=$ $A^{*} \cap R$ is equivalent to the inclusion $A^{*} \cap R \subset R^{*}$, the latter being actually a formulation of condition (ii).

(ii) $\Rightarrow$ (iii) Assume that (ii) holds and consider elements $a, b \in R$ relatively prime in $A$. If $c$ is a common divisor of $a$ and $b$ in $R$, then it is obviously their common divisor in $A$. Hence $c$ is invertible in $A$, and then by (ii) it is invertible in $R$. Consequently, $a$ and $b$ are relatively prime in $R$.

(iii) $\Rightarrow$ (ii) It is sufficient to notice that $a \in R$ is invertible (in $A$ or $R$, respectively) if and only if it is relatively prime with 1 .

Proposition 1.3. Let $A$ be a domain. Let $R$ be a subring of $A$. Consider the following conditions:

(i) for every $a, b \in R$, if $\left.a\right|_{A} b$, then $\left.a\right|_{R} b$,

(ii) for every $a, b \in R$, if $a \sim_{A} b$, then $a \sim_{R} b$,

(iii) for every $a, b \in R$, if $a \operatorname{rpr}_{A} b$, then $a \operatorname{rpr}_{R} b$.

(iv) for every $a \in R$, if $a \in \operatorname{Irr} A$, then $a \in \operatorname{Irr} R$.

Then

$$
\text { (i) } \Rightarrow \text { (ii) } \Rightarrow \text { (iii) } \Rightarrow \text { (iv). }
$$

Proof. (i) $\Rightarrow$ (ii) It suffices to note that $a, b \in R$ are associated (in $A$ or $R$, respectively) if and only if $a \mid b$ and $b \mid a$.

(ii) $\Rightarrow$ (iii) It suffices to observe that $a \in R$ is invertible (in $A$ or $R$, respectively) if and only if it is associated with 1 . Then the assertion follows from the equivalence (ii) $\Leftrightarrow$ (iii) in Proposition 1.2 .

(iii) $\Rightarrow$ (iv) Assume that (iii) holds and consider $a \in R$ reducible in $R$. Then $a=b c$ for some elements $b, c \in R$ not invertible in $R$. From (iii) we deduce that $b$ and $c$ are not invertible in $A$ (see Proposition 1.2 ), hence $a$ is reducible in $A$.

As a consequence of Propositions 1.1, 1.2 and 1.3 , we obtain Corollary 1.4 .

Corollary 1.4. If $A$ is a domain and $R$ is a subring of $A$, then the following implications hold:

$$
\begin{aligned}
& R_{0} \cap A=R \\
& R^{*}=A^{*} \stackrel{\Downarrow}{\ngtr} A^{*} \cap R=R^{*} \Rightarrow R \cap \operatorname{Irr} A \subset \operatorname{Irr} R .
\end{aligned}
$$

It is easily seen that none of the one-way implications of Proposition 1.3 can be reversed in general.

EXAMPLE 1.5. If $A$ is equal to $k[x]$, a polynomial ring in one variable over a field $k$, and $R=k\left[x^{2}, x^{3}\right]$, then condition (i) is not fulfilled, because $x^{3}$ is 
divisible by $x^{2}$ in $A$, but it is not in $R$. However (ii) holds, because $A^{*}=k \backslash\{0\}$, hence if $f$ and $g$ are associated in $A$, then $f=c g$ for some $c \in k \backslash\{0\}$, that is, $f$ and $g$ are associated in $R$.

ExAmple 1.6. If $A=k(x)[y]$ and $R=k[x y, y]$, where $k$ is a field, then condition (ii) does not hold, because $x y$ and $y$ are associated in $A$, yet they are not in $R$. Condition (iii) is fulfilled, since $A^{*} \cap R=k(x) \cap k[x y, y]=k=R^{*}$ (see Proposition 1.2).

ExAmple 1.7. If $A$ is a field and $R$ is not a field, then (iii) is not fulfilled, because an irreducible element of $R$ is not relatively prime with 1 in $R$, but is relatively prime with 1 in $A$. Condition (iv) holds, since $\operatorname{Irr} A=\emptyset$.

It is worth noting that the conditions in Corollary 1.4 have no immediate relationship with unique factorization in $R$. More precisely, the strongest conditions in Corollary 1.4 do not imply unique factorization in $R$, and unique factorization in $R$ does not imply the weakest of the conditions in question. Here are examples.

ExAmple 1.8. Let $A=k[x, y]$ and $R=k\left[x^{2}, y^{2}, x y\right]$, where $k$ is a field. Then both conditions $R^{*}=A^{*}$ and $R_{0} \cap A=R$ are fulfilled, but there is no unique factorization in $R$, since $x^{2} \cdot y^{2}=(x y)^{2}$.

ExAmple 1.9. Let $A=k(x)[y]$ and $R=k[x, y]$, where $k$ is a field. Clearly $R$ is a unique factorization domain, but $R \cap \operatorname{Irr} A \subset \operatorname{Irr} R$ does not hold, because $x y$ is irreducible in $A$ and reducible in $R$.

2. Factoriality with respect to a subring. We introduce the notion of factorial closedness of one subring with respect to factors from another subring.

Definition 2.1. Let $B$ be a subring of $A$. The subring $R$ of $A$ is called $B$-factorially closed, if, whenever $a \in A, b \in B$ and $a b \in R \backslash\{0\}$, then $a \in R$. If $R$ is $R$-factorially closed, then we call it self-factorially closed.

Note that " $A$-factorially closed" in the sense of the above definition is equivalent to the usual notion of "factorially closed" (in $A$ ).

Now, let $A$ be a domain of characteristic $p>0$ and let $R$ be a subring of $A$. An element $a \in A$ is called separably algebraic over $R$ if $w(a)=0$ for some irreducible polynomial $w(T) \in R_{0}[T] \backslash R_{0}\left[T^{p}\right]$, that is, $a$ is separably algebraic over the subfield $R_{0}$ as an element of the field $A_{0}$ (see $[\mathbf{1 3}$, Definition 2.1). The subring $R$ is called separably algebraically closed in $A$ if all elements of $A$ separably algebraic over $R$ belong to $R$.

Proposition 2.2. Let $A$ be a domain of characteristic $p>0$ and let $R$ be a subring of $A$ such that $A^{p} \subset R$, where $A^{p}=\left\{a^{p}, a \in A\right\}$. Then the following conditions are equivalent: 
(i) the ring $R$ is separably algebraically closed in $A$,

(ii) $R_{0} \cap A=R$,

(iii) the ring $R$ is self-factorially closed in $A$,

(iv) the ring $R$ is $A^{p}$-factorially closed in $A$.

Proof. (i) $\Leftrightarrow$ (ii) was stated in $[\mathbf{1 3}$, Proposition 2.2,

(ii) $\Leftrightarrow$ (iii) follows from Lemma 1.1 ,

(iii) $\Rightarrow$ (iv) is obvious.

(iv) $\Rightarrow$ (iii) Assume that condition (iv) holds and consider $a \in A$ and $b \in R$ such that $a b \in R \backslash\{0\}$. Then $a b^{p} \in R \backslash\{0\}$ so, by the assumption, $a \in R$.

If $R$ is a finitely generated $K$-algebra such that $A^{p} \subset R$, then the above equivalent conditions characterize $R$ as a ring of constants of some $K$-derivation of $A$.

3. A general diagram of implications. In this section we consider various properties similar to $\operatorname{Irr} R \subset \operatorname{Sqf} A$ and $\operatorname{Sqf} R \subset \operatorname{Sqf} A$, and we present basic relations between them.

Given a ring $R$, we denote the following sets:

- Prime $R$ of all prime elements of $R$,

- Gpr $R$ of (single) generators of principal radical ideals of $R$,

- Rdl $R$ of radical ideals of $R$ (see $[\mathbf{3}$, p. 67 ).

LEMMA 3.1. If $R$ is a ring, then:

a) $\operatorname{Irr} R \subset \operatorname{Sqf} R$,

b) Prime $R \subset$ Gpr $R$.

Proof. a) Consider an element $x \in R$. Assume that $x \notin$ Sqf $R$, that is $x=y^{2} z$, where $y \in R \backslash R^{*}, z \in R$. Then $x=y \cdot(y z)$, where $y, y z \in R \backslash R^{*}$, so $x \notin \operatorname{Irr} R$.

b) This holds because every prime ideal is radical.

LEMMA 3.2. If $R$ is a domain, then:

a) Prime $R \subset \operatorname{Irr} R$,

b) $\operatorname{Gpr} R \subset \operatorname{Sqf} R$.

Proof. a) This fact is well known.

b) Consider an element $r \in \operatorname{Gpr} R$. Let $r=x^{2} y$, where $x, y \in R$. We have $(x y)^{2}=r y$, so $(x y)^{2} \in R r$, and then $x y \in R r$, because $R r$ is a radical ideal. We obtain $x y=r z$, so $x y=x^{2} y z$, and hence $1=x z$.

From these two lemmas we obtain the following proposition. 
Proposition 3.3. Let $A$ be a domain. Let $R$ be a subring of $A$. Then the following implications hold:

$\operatorname{Irr} R \subset \operatorname{Irr} A \Rightarrow$ Prime $R \subset \operatorname{Irr} A \Leftarrow \operatorname{Prime} R \subset \operatorname{Prime} A \Leftarrow \forall_{I \in \operatorname{Spec} R} A I \in \operatorname{Spec} A$

$\Downarrow \quad \Downarrow \quad \Downarrow \quad \forall$

$\operatorname{Irr} R \subset \operatorname{Sqf} A \Rightarrow$ Prime $R \subset \operatorname{Sqf} A \Leftarrow \operatorname{Prime} R \subset \operatorname{Gpr} A \Leftarrow \forall_{I \in \operatorname{Spec} R} A I \in \operatorname{Rdl} A$

Sqf $R \stackrel{\Uparrow}{\subset} \operatorname{Sqf} A \Rightarrow \operatorname{Gpr} R \subset \operatorname{Sqf} A \Leftarrow \operatorname{Gpr} R \stackrel{\Uparrow}{\subset} \operatorname{Gpr} A \Leftarrow \forall_{I \in \operatorname{Rdl} R} A I \in \operatorname{Rdl} A$

4. Some factorial conditions for subrings. The last section contains various properties in a factorial form. In the first proposition we express a condition from [17], Theorem 3.4 in terms of irreducible and square-free factorizations.

Proposition 4.1. Let $A$ be a UFD and let $R$ be a subring of $A$ such that $R^{*}=A^{*}$. Then the following conditions are equivalent:

(i) $\forall a \in A, \forall b \in \operatorname{Sqf} A, a^{2} b \in R \backslash\{0\} \Rightarrow a, b \in R$,

(ii) $\forall s_{0}, \ldots, s_{n} \in \operatorname{Sqf} A, s_{n}^{2^{n}} \ldots s_{1}^{2} s_{0} \in R \Rightarrow s_{0}, \ldots, s_{n} \in R$,

(iii) $\forall q_{1}, \ldots, q_{n} \in \operatorname{Irr} A, q_{i} \varkappa_{A} q_{j}, i \neq j, \forall k_{1}, \ldots, k_{n} \geqslant 0$

$$
q_{1}^{k_{1}} \ldots q_{n}^{k_{n}} \in R \Rightarrow \forall i, q_{1}^{c_{i}^{(1)}} \ldots q_{n}^{c_{i}^{(n)}} \in R
$$

where $k_{j}=c_{r}^{(j)} 2^{r}+\ldots+c_{0}^{(j)} 2^{0}$ for $j=1, \ldots, n$, and $c_{i}^{(j)} \in\{0,1\}$ for $i=0, \ldots, r$.

Proof. (ii) $\Rightarrow$ (i) Assume (ii) and consider elements $a \in A, b \in \operatorname{Sqf} A$. Let $a=s_{n}^{2^{n}} \ldots s_{1}^{2} s_{0}$, where $s_{0}, \ldots, s_{n} \in$ Sqf $A$. If $a^{2} b=s_{n}^{2^{n+1}} \ldots s_{1}^{2^{2}} s_{0}^{2} b \in R \backslash\{0\}$, then $s_{n}, \ldots, s_{1}, s_{0}, b \in R$ by (ii), and then $a=s_{n}^{2} \ldots s_{1}^{2} s_{0} \in R$.

(iii) $\Rightarrow$ (ii) Assume condition (iii). Let $s_{0}, \ldots, s_{n} \in \operatorname{Sqf} A$ satisfy $s_{n}^{2^{n}} \ldots s_{1}^{2} s_{0} \in$ $R$. We can write $s_{i}=u_{i} q_{1}^{c_{i}^{(1)}} \ldots q_{m}^{c_{i}^{(m)}}$, where $u_{i} \in A^{*}, q_{1}, \ldots, q_{m} \in \operatorname{Irr} A$ with $q_{j} \varkappa_{A} q_{l}$ for $j \neq l$ and $c_{i}^{(j)} \in\{0,1\}$. Then $s_{n}^{2^{n}} \ldots s_{1}^{2} s_{0}=u_{n}^{2^{n}} \ldots u_{1}^{2} u_{0} \cdot q_{1}^{k_{1}} \ldots q_{m}^{k_{m}}$, where $k_{j}=c_{n}^{(j)} 2^{n}+\ldots+c_{1}^{(j)} 2+c_{0}^{(j)}$. By the assumption, if $q_{1}^{k_{1}} \ldots q_{m}^{k_{m}} \in R$, then $q_{1}^{c_{i}^{(1)}} \ldots q_{m}^{c_{i}^{(m)}} \in R$ for $i=1,2, \ldots, n$. Hence $s_{0}, \ldots, s_{n} \in R$.

(ii) $\Rightarrow$ (iii) Assume (ii). Let $q_{1}^{k_{1}} \ldots q_{n}^{k_{n}} \in R$, where $q_{1}, \ldots, q_{n} \in \operatorname{Irr} A, q_{j} \chi_{A} q_{l}$ for $j \neq l$. Put $k_{j}=c_{r}^{(j)} 2^{r}+\ldots+c_{1}^{(j)} 2+c_{0}^{(j)}$ for $j=1, \ldots, n$, where $c_{i}^{(j)} \in\{0,1\}$. Let $s_{i}=q_{1}^{c_{i}^{(1)}} \ldots q_{n}^{c_{i}^{(n)}}$. By the assumption, since $s_{n}^{2^{n}} \ldots s_{1}^{2} s_{0} \in R$, we obtain $s_{0}, \ldots, s_{n} \in R$.

(i) $\Rightarrow$ (ii) Simple induction.

Note that factorial closedness can be expressed in the following way. 
Proposition 4.2. Let $A$ be a UFD and let $R$ be a subring of $A$ such that $R^{*}=A^{*}$. Then the following conditions are equivalent:

(i) $\forall a, b \in A, a b \in R \backslash\{0\} \Rightarrow a, b \in R$,

(ii) $\forall q_{1}, \ldots, q_{n} \in \operatorname{Irr} A, \forall k_{1}, \ldots, k_{n} \geqslant 1, q_{1}^{k_{1}} \ldots q_{n}^{k_{n}} \in R \Rightarrow q_{1}, \ldots, q_{n} \in R$.

In the next two propositions we consider factorizations with respect to relatively prime elements.

Proposition 4.3. Let $A$ be a UFD and let $R$ be a subring of $A$ such that $R^{*}=A^{*}$. Then the following conditions are equivalent:

(i) $\forall a, b \in A, a \operatorname{rpr}_{A} b, a b \in R \backslash\{0\} \Rightarrow a, b \in R$,

(ii) $\forall a_{1}, \ldots, a_{n} \in A, a_{i} \operatorname{rpr}_{A} a_{j}, i \neq j, a_{1} \ldots a_{n} \in R \backslash\{0\} \Rightarrow a_{1}, \ldots, a_{n} \in R$,

(iii) $\forall q_{1}, \ldots, q_{n} \in \operatorname{Irr} A, q_{i} \chi_{A} q_{j}, i \neq j, \forall k_{1}, \ldots, k_{n} \geqslant 1$,

$$
q_{1}^{k_{1}} \ldots q_{n}^{k_{n}} \in R \Rightarrow q_{1}^{k_{1}}, \ldots, q_{n}^{k_{n}} \in R .
$$

Proof. We see that (i) and (iii) are special cases of (ii).

(i) $\Rightarrow$ (ii) Simple induction.

(iii) $\Rightarrow$ (i) Assume (iii). Consider $a, b \in A, a \operatorname{rpr}_{A} b$, such that $a b \in R \backslash\{0\}$. Put $a=u q_{1}^{k_{1}} \ldots q_{s}^{k_{s}}$ and $b=v q_{s+1}^{k_{s+1}} \ldots q_{n}^{k_{n}}$, where $u, v \in A^{*}, q_{1}, \ldots, q_{n} \in \operatorname{Irr} A$, $q_{i} \chi_{A} q_{j}$ for $i \neq j$. By the assumption, since $a b=u v q_{1}^{k_{1}} \ldots q_{s}^{k_{s}} q_{s+1}^{k_{s+1}} \ldots q_{n}^{k_{n}} \in R$, we have $q_{1}^{k_{1}}, \ldots, q_{s}^{k_{s}}, q_{s+1}^{k_{s+1}}, \ldots, q_{n}^{k_{n}} \in R$. Finally, $a=u q_{1}^{k_{1}} \ldots q_{s}^{k_{s}} \in R$ and $b=v q_{s+1}^{k_{s+1}} \ldots q_{n}^{k_{n}} \in R$.

Proposition 4.4. Let $A$ be a UFD and let $R$ be a subring of $A$ such that $R^{*}=A^{*}$. Then the following conditions are equivalent:

(i) $\forall a, b \in A, a \operatorname{rpr}_{A} b, \forall k>1,\left(a^{k} b \in R \backslash\{0\} \Rightarrow a, b \in R\right)$,

(ii) $\forall q_{1}, \ldots, q_{n} \in \operatorname{Irr} A, q_{i} \nsim_{A} q_{j}, i \neq j, \forall k_{1}, \ldots, k_{r}>1, r \leqslant n$,

$$
q_{1}^{k_{1}} \ldots q_{r}^{k_{r}} q_{r+1} \ldots q_{n} \in R \Rightarrow q_{1}, \ldots, q_{r}, q_{r+1} \ldots q_{n} \in R .
$$

Proof. (i) $\Rightarrow$ (ii) Assume (i). Let $q_{1}^{k_{1}} \ldots q_{r}^{k_{r}} q_{r+1} \ldots q_{n} \in R$ for some pairwise non-associated $q_{1}, \ldots, q_{n} \in \operatorname{Irr} A$ and for some $k_{1}, \ldots, k_{r}>1$. By the assumption we have $q_{1} \in R$ and $q_{2}^{k_{2}} \ldots q_{r}^{k_{r}} q_{r+1} \ldots q_{n} \in R$, then $q_{2} \in R$ and $q_{3}^{k_{3}} \ldots q_{r}^{k_{r}} q_{r+1} \ldots q_{n} \in R$, and so on, until we obtain $q_{r} \in R$ and $q_{r+1} \ldots q_{n} \in R$. (ii) $\Rightarrow$ (i) Assume (ii). Consider $a, b \in A$ such that $a \operatorname{rpr}_{A} b$ and $a^{k} b \in R \backslash\{0\}$ for some $k>1$. We can write $a=u q_{1}^{l_{1}} \ldots q_{r}^{l_{r}}$ and $b=v q_{r+1}^{l_{r+1}} \ldots q_{s}^{l_{s}} q_{s+1} \ldots q_{n}$, where $u, v \in A^{*}, q_{1}, \ldots, q_{n} \in \operatorname{Irr} A, q_{i} \chi_{A} q_{j}$ for $i \neq j$ and $l_{r+1}, \ldots, l_{s}>1$. We have: $a^{k} b=u^{k} v\left(q_{1}^{l_{1}} \ldots q_{r}^{l_{r}}\right)^{k} q_{r+1}^{l_{r+1}} \ldots q_{s}^{l_{s}} q_{s+1} \ldots q_{n} \in R$. Then $q_{1}, q_{2}, \ldots, q_{s}$, $q_{s+1} \ldots q_{n} \in R$. Finally, $a, b \in R$.

The following proposition shows that if we omit the restriction $b \in \operatorname{Sqf} R$ in Proposition 4.1, then we obtain usual factorial closedness. 
Proposition 4.5. Let $A$ be a domain. Let $R$ be a subring of $A$. Then the following conditions are equivalent:

(i) $\forall a, b \in A, a b \in R \backslash\{0\} \Rightarrow a, b \in R$,

(ii) $\forall a, b \in A, a^{2} b \in R \backslash\{0\} \Rightarrow a, b \in R$,

(iii) $\forall a, b \in A, a^{3} b \in R \backslash\{0\} \Rightarrow a, b \in R$,

(iv) $\forall a, b \in A,\left(a^{2} b \in R \backslash\{0\} \vee a^{3} b \in R \backslash\{0\}\right) \Rightarrow a, b \in R$,

(v) $\forall a, b \in A, \forall k>1\left(a^{k} b \in R \backslash\{0\} \Rightarrow a, b \in R\right)$,

(vi) $\forall a, b \in A, \forall k \geqslant 1\left(a^{k} b \in R \backslash\{0\} \Rightarrow a, b \in R\right)$.

Proof. (ii) $\Rightarrow$ (i) Assume that (ii) holds. Let $a b \in R \backslash\{0\}$ for some $a, b \in A$. Then $a^{2} b^{2} \in R \backslash\{0\}$. By the assumption we have $a, b^{2} \in R$. Again using assumption for $b^{2} \cdot 1 \in R$ we have $b \in R$. Finally, $a, b \in R$.

(iii) $\Rightarrow$ (i) Assume that (iii) holds. Let $a b \in R \backslash\{0\}$ for some $a, b \in A$. Then $a^{3} b^{3} \in R \backslash\{0\}$. By the assumption we have $a, b^{3} \in R$. Again using assumption for $b^{3} \cdot 1$ we have $b \in R$. Finally, $a, b \in R$.

Implications: (vi) $\Rightarrow$ (v), (v) $\Rightarrow$ (iv), (iv) $\Rightarrow$ (ii), (iv) $\Rightarrow$ (iii) are obvious.

(i) $\Rightarrow$ (vi) Simple induction.

The last proposition is motivated by a modification of Proposition 4.5

Proposition 4.6. Let $A$ be a domain. Let $R$ be a subring of $A$. Then the following conditions are equivalent:

(i) $\forall a, b \in A, a b, a^{2} b \in R \backslash\{0\} \Rightarrow a, b \in R$,

(ii) $\forall a, b \in A, a^{2} b, a^{3} b \in R \backslash\{0\} \Rightarrow a, b \in R$,

(iii) $\forall a, b \in A\left(\forall k \geqslant 1 a^{k} b \in R \backslash\{0\}\right) \Rightarrow a, b \in R$,

(iv) $\forall a, b \in A\left(\forall k>1 a^{k} b \in R \backslash\{0\}\right) \Rightarrow a, b \in R$,

(v) $\forall a, b \in A\left(\exists k_{0}, \forall k \geqslant k_{0}, a^{k} b \in R \backslash\{0\}\right) \Rightarrow a, b \in R$.

Proof. (iii) $\Rightarrow$ (i), (ii), (v) Assume that condition (iii) holds. It is enough to prove that if $a^{k} b, a^{k+1} b \in R \backslash\{0\}$ for some $a, b \in A$, then $a^{k+2} b \in R \backslash\{0\}$ and (if $k>1$ ) $a^{k-1} b \in R \backslash\{0\}$.

Assume that $a^{k} b, a^{k+1} b \in R \backslash\{0\}$, where $a, b \in A$. Since

$$
\left(a^{k} b\right)^{l} \cdot a^{k+2} b=\left(a^{k+1} b\right)^{2} \cdot\left(a^{k} b\right)^{l-1} \in R
$$

holds for every $l \geqslant 1$, from the assumption we obtain $a^{k+2} b \in R$. Moreover, if $k>1$, since

$$
\left(a^{k+1} b\right)^{l} \cdot a^{k-1} b=\left(a^{k} b\right)^{2} \cdot\left(a^{k+1} b\right)^{l-1} \in R
$$

also holds for every $l \geqslant 1$, we infer also $a^{k-1} b \in R$.

Implications: (v) $\Rightarrow$ (iv), (iv) $\Rightarrow$ (iii), (i) $\Rightarrow$ (iii), (ii) $\Rightarrow$ (iv) are obvious. 


\section{References}

1. Anderson D.F., Root closure in integral domains, J. Algebra, 79 (1982), 51-59.

2. Ayad M., Sur les polynômes $f(X, Y)$ tels que $K[f]$ est intégralement fermé dans $K[X, Y]$, Acta Arith., 105 (2002), 9-28.

3. Belluce L.P., Di Niola A., Ferraioli A.R., Ideals of $M V$-semirings and $M V$-algebras, in: G.L. Litvinov, S.N. Sergeev (eds), Tropical and idempotent mathematics and applications, AMS, Providence, 2014.

4. Bondt M. de, Yan D., Irreducibility properties of Keller maps, Algebra Colloq., 23 (2016), 663-680.

5. Brewer J.W., Costa D.L., McCrimmon K., Seminormality and root closure in polynomial rings and algebraic curves, J. Algebra, 58 (1979), 217-226.

6. Daigle D., Locally nilpotent derivations, Lecture notes for the "September School" of Algebraic Geometry, Łukęcin, Poland, September 2003 (unpublished), aix1.uottawa.ca/ ddaigle/.

7. Essen A. van den, Polynomial automorphisms and the Jacobian Conjecture, Birkhäuser Verlag, Basel, 2000.

8. Freudenburg G., Algebraic theory of locally nilpotent derivations, Springer Verlag, Berlin, 2006.

9. Geroldinger A., Halter-Koch F., Non-unique factorizations, algebraic, combinatorial and analytic theory, Chapman \& Hall/CRC, Boca Raton, 2006.

10. Jędrzejewicz P., Rings of constants of p-homogeneous polynomial derivations, Comm. Algebra, 31 (2003), 5501-5511.

11. Jędrzejewicz P., Eigenvector p-bases of rings of constants of derivations, Comm. Algebra, 36 (2008), 1500-1508.

12. Jędrzejewicz P., One-element p-bases of rings of constants of derivations, Osaka J. Math., 46 (2009), 223-234.

13. Jędrzejewicz P., A note on rings of constants of derivations in integral domains, Colloq. Math., 122 (2011), 241-245.

14. P. Jędrzejewicz, Positive characteristic analogs of closed polynomials, Cent. Eur. J. Math., 9 (2011), 50-56.

15. Jędrzejewicz P., Jacobian conditions for p-bases, Comm. Algebra, 40 (2012), 2841-2852.

16. Jędrzejewicz P., A characterization of Keller maps, J. Pure Appl. Algebra, 217 (2013), $165-171$.

17. Jędrzejewicz P., Zieliński J., Analogs of Jacobian conditions for subrings, to appear in J. Pure Appl. Algebra, arXiv:1601.01508.

18. Nowicki A., Polynomial derivations and their rings of constants, Nicolaus Copernicus University, Torun, 1994, www.mat.umk.pl/ anow/.

19. Nowicki A., Rings and fields of constants for derivations in characteristic zero, J. Pure Appl. Algebra, 96 (1994), 47-55.

Received October 6, 2016

Faculty of Mathematics and Computer Science Nicolaus Copernicus University

ul. Chopina $12 / 18$

87-100 Toruń

Poland

e-mail: pjedrzej@mat.umk.pl

e-mail: lmatysiak@mat.umk.pl

e-mail: ubukrool@mat.umk.pl 